\title{
Hacia el alineamiento global \\ en la armonización regional: \\ la regulación de los aditivos alimentarios \\ en el Mercosur*
}

\author{
Towards the global alignment \\ in the regional harmonization: \\ the Mercosur food additive regulation
}

SUMARIO

I. La regulación alimentaria global. I.1. Los Acuerdos de la omc. I.2. El Codex Alimentarius. I.3. Armonización regional de la regulación alimentaria: las resoluciones del Grupo Mercado Común (Mercosur). II. La regulación de los aditivos alimentarios. II.1. El alineamiento regional tras los estándares globales. II.2. Una excepción a la regla de alineamiento tras los estándares globales: las especies botánicas de origen regional. Conclusiones.

RESUMEN

Este trabajo pretende analizar el modo en que la normativa internacional de agencias globales como la FAO, la OMC y la OMS limita y condiciona las posibilidades de una construcción autónoma de la regulación regional sobre alimentos. Esto se muestra a partir del análisis de un caso: el proceso de autorización de los aditivos alimentarios en el Mercosur. En primer lugar, se mapea brevemente la regulación alimentaria a nivel global, ante todo los Acuerdos de la omc en la materia y las normas del CodEX AlimentARIUs (FAO/ OMS). Se trata de mostrar su influencia en la armonización legislativa alimen-

* Recibido el 10 de octubre de 2014, aprobado el 11 de febrero de 2015.

Para citar el artículo: M. E. MARICHAL. Hacia el alineamiento global en la armonización regional: la regulación de los aditivos alimentarios en el Mercosur. Revista Derecho del Estado n. ${ }^{\circ}$ 34, Universidad Externado de Colombia, enero-junio de 2015, pp. 183-202. DOI: 10.18601/01229893.n34.09

** Abogada de la Universidad Nacional del Litoral. Magíster en Ciencia, Tecnología y Sociedad por la Universidad Nacional de Quilmes (Argentina). Docente e Investigadora del Centro de Investigaciones de la Facultad de Ciencias Jurídicas y Sociales de la Universidad Nacional del Litoral. Candidata a doctora en Derecho por la Universidad Nacional del Litoral (Santa Fe, Argentina).Contacto: marichal@fcjs.unl.edu.ar 
taria en la región del Mercosur que tiene lugar a través de las resoluciones del Grupo Mercado Común (GMC). Segundo, se explica el caso analizado (la autorización de los aditivos alimentarios) describiendo el modo en que la normativa regional genera un efecto de alineamiento en función de estándares producidos al exterior de la región. Sin embargo, esta normativa trae una excepción a la regla de alineamiento general (las especies botánicas de origen regional) que nos permite vislumbrar una forma alternativa de regulación emancipatoria.

PALABRAS CLAVE

Mercosur, integración regional, derecho internacional, legislación alimentaria, aditivo alimentario.

\section{ABSTRACT}

This paper aims to analyze the way in which international regulation of global agencies such as FAO, wTO and wHO limits and conditions the possibilities of an autonomous making process of the regional food law. This is shown from a particular case study: the food additive authorization process in Mercosur. First, the paper presents a brief global food law mapping, mainly the WTO Agreements regarding food law. We try to demonstrate its influence in the Mercosur region food law harmonization that take place through the Grupo Mercado Comun resolutions. Second, the case study is explained (food additives authorization) describing the way in which the regional normative produce an alignment effect behind externally produced standards. Nevertheless, this normative brings a rule exception (the regional origin botanical species) that allows us to envisage an alternative way of emancipatory regulation.

\section{KEYWORDS}

Mercosur, regional integration, international law, foodstuffs legislation, food additive.

\section{LA REGULACIÓN ALIMENTARIA GLOBAL}

Las cuestiones vinculadas a los alimentos constituyen uno de los sectores sociales y económicos más regulados, al punto de conformar una miríada de reglas globales, regionales, nacionales y locales, tanto del ámbito público como privado. En la "arena global", poderosas organizaciones, tales como la ONU, la FAO, la Comisión del Codex Alimentarius y la OMC, juegan importantes roles en la gobernanza global de los alimentos mediante la formulación 
e implementación de reglas sobre la producción, manufactura, comercio, transporte y distribución de estos bienes ${ }^{1}$.

El organismo internacional que marca los lineamientos de la regulación del comercio internacional de los alimentos es, sin duda, la Organización Mundial del Comercio (OMC) ${ }^{2}$. Esta organización se crea para brindar un espacio para negociar acuerdos comerciales internacionales. Es el foro al que los gobiernos de sus Estados Miembros recurren tanto para regular las relaciones comerciales internacionales como para resolver los problemas que puedan suscitarse ${ }^{3}$.

La labor de la OMC se orienta hacia la remoción de los obstáculos al comercio internacional a través de la armonización de la regulación de bienes comerciales. Oficialmente se expresa que este objetivo central se conjuga con otros intereses, tales como la protección de los consumidores o el impedimento de la propagación de enfermedades. Sin embargo, la afectación a estos otros intereses no comerciales es tratada como un "efecto secundario" de las medidas de remoción de obstáculos al comercio que, en principio, deberían ser tolerados ${ }^{4}$.

Las reglas de este comercio internacional y del funcionamiento de la OMC se concentran en una serie de acuerdos que son negociados y firmados por la mayoría de los países miembros ${ }^{5}$. Se han firmado dos acuerdos relativos

1 Bernd van der Meulen, "The Global Arena of Food Law: Emerging Contours of a Meta-Framework", Erasmus Law Review, 3 (2010), 217-240.

2 La OMC fue el resultado de negociaciones sostenidas entre 1986-1994 en la Ronda Uruguay (que concluyó en Marrakech) y de anteriores negociaciones en el marco del Acuerdo General sobre Aranceles Aduaneros y Comercio (General Agreement on Tariffs and Trade-GATT). La Ronda Uruguay (que incluyó la negociación de medidas sobre bienes, servicios y propiedad intelectual) significó la aplicación, por primera vez, de normas comerciales internacionales a los productos agrícolas. FAO, Directrices En Materia de Legislación Alimentaria (nuevo Modelo de Ley de Alimentos Para Países de Tradición Jurídica Romano-Germánica), Estudio Legislativo (FAO, 2006).

3 La omC se compone de ciento cincuenta y nueve miembros (a 12 de marzo de 2014). Todos los países miembros del Mercosur (Argentina, Brasil, Uruguay, Paraguay, Bolivia y Venezuela) forman parte de la organización desde su inicio en el año 1995. [http://www.wto.org/ spanish/thewto_s/whatis_s/tif_s/org6_s.htm]. Acceso: 15/04/2014

4 El propósito primordial del sistema es contribuir a que el comercio internacional fluya con la mayor libertad posible sin que se produzcan "efectos secundarios no deseables, porque eso es importante para el desarrollo económico y el bienestar": [http://www.wto.org/spanish/ thewto_s/whatis_s/who_we_are_s.htm]. Acceso: 16/11/2012.

5 Estos Acuerdos son "contratos que obligan a los gobiernos a mantener sus políticas comerciales dentro de límites convenidos". Si bien formalmente son los gobiernos los que negocian en el foro, un cúmulo de intereses del mercado internacional de bienes y servicios se manifiestan a través de ellos. Desde la OMC se expresa que la finalidad última de estos acuerdos "es ayudar a los productores de bienes y servicios, a los exportadores y a los importadores a desarrollar sus actividades, si bien permitiendo que los gobiernos alcancen objetivos sociales y ambientales": http://www.wto.org/spanish/thewto_s/whatis_s/who_we_are_s.htm Acceso: 19/12/2012. Sin embargo, el sistema global de regulación de los alimentos no parece estar dirigido directamente al comportamiento de los operadores del mercado alimentario, sino más 
a la seguridad, comercio y normalización de los alimentos: el Acuerdo sobre Medidas Sanitarias y Fitosanitarias (AMSFS) y el Acuerdo de Obstáculos Técnicos al Comercio (АОтС).

\section{I.1. Los Acuerdos de la омC}

El AMSFS regula la inocuidad de los alimentos y la salubridad de animales y plantas a nivel internacional. Es considerado uno de los logros más significativos de la Ronda Uruguay que creó la $\mathrm{OMC}^{6}$. A través de este Acuerdo, la OMC reconoce a los gobiernos el derecho de adoptar las medidas sanitarias y fitosanitarias que consideren convenientes para "proteger la salud y la vida de las personas y de los animales o para preservar los vegetales, siempre que tales medidas no sean incompatibles con las disposiciones del presente Acuerdo" (art. 2.1 AMSFs). Además, los miembros deben asegurarse de que cualquier medida que adopten "sólo se aplique en cuanto sea necesaria para proteger la salud y la vida de las personas y de los animales o para preservar los vegetales, de que esté basada en principios científicos y de que no se mantenga sin testimonios científicos suficientes" (art. 2.2 AMSFS) ${ }^{7}$.

Si bien el acuerdo no obliga a los miembros a adoptar medidas sanitarias y fitosanitarias de contenido concreto, los alienta a que basen las medidas que "libremente" adopten en normas, directrices y recomendaciones internacionales, cuando estas existan, con el objetivo de facilitar la armonización de la regulación internacional ${ }^{8}$. En relación a la inocuidad alimentaria, el Acuerdo recomienda a los países miembros fundar sus medidas en la regulación de la Comisión del Codex Alimentarius ${ }^{9}$.

bien a los marcos regulatorios nacionales que tienen por objeto tales comportamientos. En este sentido, la regulación global alimentaria es un meta-marco regulatorio del sector alimentario. Cfr. Bernd van Der Meulen.

6 Steve Charnovitz, "Improving the Agreement on Sanitary and Phytosanitary Standards", en Trade, environment, and the Millenium, GARY P. SAMPSON (Overseas Development Council, 2000), p. 171.

7 Las medidas sanitarias y fitosanitarias tampoco pueden significar una "discriminación arbitraria o injustificable entre Miembros en que prevalezcan condiciones idénticas o similares, ni entre su propio territorio y el de otros Miembros. Las medidas sanitarias y fitosanitarias no se aplicarán de manera que constituyan una restricción encubierta del comercio internacional" (art. 2.3 AMSFS).

8 "Para armonizar en el mayor grado posible las medidas sanitarias y fitosanitarias, los Miembros basarán sus medidas sanitarias o fitosanitarias en normas, directrices o recomendaciones internacionales, cuando existan, salvo disposición en contrario en el presente Acuerdo y en particular en el párrafo 3" (art. 3.1 AMSFS).

9 Además del repertorio de disposiciones sobre requisitos sanitarios para el comercio internacional de los alimentos establecidos en el Codex Alimentarius, existen una variedad de estándares internacionales que los países importadores pueden exigir, como los procedimientos de verificación de la calidad de productos o procesos productivos (análisis HACCP o las normas ISO). María Angélica Larach, El Acuerdo sobre la Aplicación de Medidas Sanitarias y Fitosanitarias: 
Por otro lado, el Acuerdo sobre Obstáculos Técnicos al Comercio (АОТС) se aplica a diferentes tipos de productos, incluyendo todos los productos alimentarios y los agrícolas. Abarca las normas técnicas no comprendidas en el AMSFS (tales como los requisitos de embalaje, marcado y etiquetado, los procedimientos de prueba y certificación) y tiene por objetivo evitar que los reglamentos técnicos, normas y procedimientos de prueba y certificación generen obstáculos "innecesarios" al comercio mundial ${ }^{10}$. Con una lógica similar al AMSFS, el AOTC reconoce a los países el derecho de adoptar medidas apropiadas para salvaguardar cuestiones legítimas (protección de la salud y la vida de las personas y de los animales, preservación de los vegetales o la protección del medio ambiente) bajo ciertas condiciones: no pueden significar obstáculos innecesarios al comercio ni discriminación entre los Estados miembros. El acuerdo alienta a los países para que utilicen las normas internacionales como referencia, si existieran y fueran apropiadas. Sin embargo, a diferencia del AMSF, el АотC no aconseja organismos internacionales determinados ${ }^{11}$.

Ambos acuerdos (AMSFS y AOTC) prevén un trato diferenciado para países en desarrollo (arts. 10 AMSFS y 12 AOTC) y países "menos adelantados" (art. 10 AMSFS). Se entiende que debido a limitaciones en los recursos financieros, humanos y técnicos, estos países enfrentan serios obstáculos para "para cumplir con los objetivos en materia de salud e inocuidad alimentaria" 12 . Si bien estos países en general cuentan con legislación y reglamentos en materia sanitaria y fitosanitaria, desde los organismos internacionales se considera que muchas de sus disposiciones son "obsoletas y no concuerdan con los Acuerdos

contenido y alcance para América Latina y el Caribe, Comercio Internacional, 39 (Santiago de Chile: ONU- CEPAL, 2003). Sobre salud y vida animal y vegetal cfr. la regulación de la Oficina Internacional de Epizootias (OIE) y la Convención Internacional de Protección Fitosanitaria (CIPF). Dichos organismos regulan sus ámbitos de aplicación también elaborando códigos: cfr. Código Sanitario para los Animales Terrestres y Código Sanitario para los Animales Acuáticos.

10 "Todos los productos, comprendidos los industriales y los agropecuarios, quedarán sometidos a las disposiciones del presente Acuerdo" (art. 1.3 AOTC). Este Acuerdo se encontraba vigente como acuerdo de aplicación voluntaria desde la Ronda de Tokio (1973-1979). Con algunas ampliaciones y modificaciones en la Ronda Uruguay (1986-1994) se convirtió en un acuerdo multilateral vinculante para los Estados parte de la OMC.

11 Las medidas adoptadas se deben justificar con información científica y técnica y métodos relacionados con el procesamiento o uso final de los productos. El AOTC promueve la armonización de las normativas nacionales y para ello incluye en un anexo un "Código de Buena Conducta para la Elaboración, Adopción y Aplicación de Normas" destinado a los organismos públicos de normalización. "Código de buena conducta para la elaboración, adopción y aplicación de normas por las instituciones de normalización, abierto a la aceptación por las instituciones tanto del sector público como del sector privado", disponible en: [http://www.wto.org/spanish/ docs_s/legal_s/legal_s.htm]. Acceso: 16/11/2012.

12 Los países considerados en vías de desarrollo y menos avanzados representan aproximadamente dos tercios de la totalidad de los miembros de la OMC. FAO, Directrices en Materia de Legislación Alimentaria (nuevo Modelo de Ley de Alimentos para Países de Tradición Jurídica Romano-Germánica). 
MSF y OTC o con normas establecidas por las organizaciones internacionales correspondientes" ${ }^{13}$. Otro problema que se detecta en estos países es su incapacidad para aplicar y reforzar las medidas sanitarias y las regulaciones técnicas que sí tienen, así como la falta de condiciones (especialmente de infraestructura) para llevar a cabo análisis de riesgos acordes a los parámetros internacionalmente establecidos. Para resolver estos y otros problemas de aplicación, los acuerdos prevén que Estados miembros de la OMC proporcionen asistencia técnica y ayuda a los países en desarrollo y a los menos desarrollados, ya sea bilateralmente (a petición de un Estado miembro, mediante convenios de facilitación de asistencia técnica entre los Estados miembros, arts. 9 AMSFS y 11 Аотс) о a través de las organizaciones internacionales pertinentes ${ }^{14}$.

\section{I.2. El Codex Alimentarius}

El Codex Alimentarius nace como una recopilación ordenada de la regulación alimentaria que la comunidad científica y técnica en la materia postulaba para la identificación, elaboración, control, etc. de los alimentos ${ }^{15}$. La Comisión del Codex Alimentarius fue establecida en 1961 como un cuerpo subsidiario de la Organización Mundial de la Salud (OMS) y la FAO para la formulación de propuestas a los directores generales de la ONU y de los órganos rectores de ambas organizaciones. Al poco tiempo se agregó la finalidad de actuar como órgano de consulta para todas las cuestiones relativas a la ejecución del Programa Conjunto FAO/OMS sobre Normas Alimentarias ${ }^{16}$. La Comisión del Codex Alimentarius es considerada la "única organización internacional que reúne científicos, expertos técnicos, entes reguladores gubernamentales y organizaciones, tanto de consumidores como industriales, con el fin de establecer normas alimentarias" ${ }^{17}$. Elabora diferentes tipos de instrumentos: estándares, códigos de prácticas, directrices y otras recomendaciones.

13 FAo, Directrices en Materia de Legislación Alimentaria (nuevo Modelo de Ley de Alimentos para Países de Tradición Jurídica Romano-Germánica).

14 La ayuda puede estar orientada, entre otras cosas, a la formulación de medidas sanitarias y regulaciones técnicas, facilitando la participación en las actividades que realizan las instituciones internacionales y estableciendo la infraestructura apropiada. La asistencia puede ser a través de asesorías, créditos, donaciones, subsidios, capacitación y equipamiento.

15 E. Boutrif, "The New Role of Codex Alimentarius in the Context of wTo/sps Agreement", Food Control, 14 (2003), 81-88.

16 Este programa fue creado en 1963 para "proteger la salud de los consumidores y asegurar prácticas equitativas en el comercio de los alimentos" y "promover la coordinación de todos los trabajos sobre normas alimentarias emprendidos por las organizaciones internacionales gubernamentales y no gubernamentales" (art. 1 Estatutos de la Comisión del Codex Alimentarius) FAO, ¿Qué es el Codex Alimentarius? (Roma: FAO, 2006), en: ftp://ftp.fao.org/codex/Publications/ understanding/Understanding_ES.pdf

17 FAo, Directrices en Materia de Legislación Alimentaria (nuevo Modelo de Ley de Alimentos para Países de Tradición Jurídica Romano-Germánica). 
El trabajo interno de la Comisión del Codex Alimentarius se realiza en comités, que pueden ser de dos tipos: comités de asuntos generales o de "naturaleza horizontal" (estudian una materia o materias generales para todo tipo de alimentos) ${ }^{18}$; y comités de productos o de "naturaleza vertical" (estudian cuestiones específicas de los alimentos dentro de una misma clase) ${ }^{19}$. Estos comités pueden solicitar asesoramiento de expertos, consultando a especialistas internacionalmente reconocidos, o bien recurrir a comités de expertos independientes de la FAO y la OMS. Actualmente, entre los comités de expertos mixtos FAO/OMS más importantes puede mencionarse al Comité de Expertos en Aditivos Alimentarios (JECFA), el Comité de Expertos sobre Residuos de Plaguicidas (JMPR) y el Comité de Expertos sobre Evaluación de Riesgos Microbiológicos (JEMRA). El asesoramiento científico de estos comités mixtos de expertos, así como los resultados de las consultas de expertos ad hoc, conforman la base de las normas elaboradas por los comités del Codex ${ }^{20}$.

Desde que la Comisión del Codex Alimentarius ha sido incorporada al AMSFS como su organización de referencia, los estándares del Codex han ganado una enorme importancia internacional. De ser un cuerpo "invisible" de establecimiento de estándares, el Codex pasó a ser una poderosa agencia regulatoria global ${ }^{21}$. A la vez, este nuevo estatus desató un cuestionamiento de la legitimidad del proceso decisorio al interior del Codex y un reclamo por estándares de responsabilidad más altos y confiables ${ }^{22}$.

18 Por ejemplo: Comité del Codex sobre Contaminantes de los Alimentos, Comité del Codex sobre Aditivos Alimentarios, Comité del Codex sobre Residuos de Plaguicidas, etc. Cfr.: [http://www.codexalimentarius.org/committees-\&-task-forces/es/]. Acceso: 17/12/2012.

19 Por ejemplo: Comité del Codex sobre Pescado y Productos Pesqueros, Comité del Codex sobre Frutas y Hortalizas Frescas, Comité del Codex sobre Azúcares, etc. Cfr.: [http:// www.codexalimentarius.org/committees-\&-task-forces/es/]. Acceso: 17/12/2012. Debido a un reciente cambio de orientación del Codex, "que va de las inquietudes relativas a la calidad hacia la inocuidad de los alimentos y la protección de la salud", se procura atender mayormente el tratamiento de los temas "horizontales" (higiene, etiquetado, aditivos y contaminantes). Esto ha incrementado el número y la importancia de los Comités de Asuntos Generales.

20 “... estos comités de expertos y consultas no son parte de la Comisión del Codex Alimentarius, pero son entidades autónomas, establecidos por la FAO y la oms para proporcionar asesoramiento especializado a la Comisión del Codex Alimentarius y sus órganos subsidiarios, así como a los gobiernos miembros": [http://www.codexalimentarius.org/bases-cientificas-para-el-codex/es/]. Acceso: 17/12/2012. Los comités y grupos de expertos están compuestos por personalidades invitadas en función de su capacidad personal y no como representantes de las instituciones a las que pertenecen o a sus gobiernos. Cfr. BoutRIF.

21 David E. Winickoff and Douglas M. Bushey, "Science and Power in Global Food Regulation: The Rise of the Codex Alimentarius", Science, Technology, \& Human Values, 35 (2010), 356-381.

22 Michel Livermore, "Authority and Legitimacy in Global Governance: Deliberation, Institutional Differentiation, and the Codex Alimentarius", New York University Law Review, 81 (2006), 766-801; Frode Veggeland and Svein Borgen, "Negotiating International Food Standards: The World Trade Organization's Impact on the Codex Alimentarius Commission", Governance, 18 (2005), 675-708. 
Aunque la conformidad con los estándares del Codex no es, en principio, una imposición obligatoria para los Estados miembros de la omc, la mayoría de los académicos coinciden en señalar que los estándares sentados por la Comisión del Codex Alimentarius se han convertido en puntos de referencia fundamentales de los requerimientos nacionales ${ }^{23}$.

\section{I.3. Armonización regional de la regulación alimentaria: las resoluciones del Grupo Mercado Común (Mercosur)}

Debido a su influencia directa e indirecta en el establecimiento de las regulaciones alimentarias nacionales (y regionales), el poder de alineamiento del Codex Alimentarius de FAO/OMS es altamente significativo ${ }^{24}$. ¿Existen tendencias o fuerzas en tensión con este proceso de alineamiento global? $\mathrm{Al}$ describir las limitaciones inherentes al marco regulatorio europeo actual, algunos autores han detectado una tensión entre dos tipos de restricciones a la autonomía regulatoria en materia de seguridad alimentaria: la mencionada limitación impuesta por los marcos globales y las restricciones que resultan de acuerdos comerciales bilaterales o regionale ${ }^{25}$. En nuestra región, un importante cauce de convergencia de las regulaciones nacionales sobre alimentos está dado por las normas producidas en el ámbito del Mercosur. Las decisiones adoptadas en el marco de este proceso de armonización regional constituyen referentes para el intercambio de bienes entre los países miembros y asociados, así como con otros países o bloques regionales.

23 Mark Mansour, "One World for All: International Harmonization of Food Regulations. Codex Alimentarius and the Treatment of Foods Deriver from Biotechnology as a Case Study", Journal of Food Science, 69 (2004); DAvid Jukes, "The Role of Science in International Food Standards", Food Control, 11 (2000), 181-194; BoutrIF D. WIRTH, "The Role of Science in the Uruguay Round and NAFTA Trade Disciplines", Cornell International Law Journal, 27 (1994), 817-859.

24 Los estándares del Codex ejercen una influencia directa cuando los Estados miembros optan por seguir la recomendación de la OMC y basan sus medidas en los estándares internacionales. Tienen una influencia indirecta como puntos de referencia cuando los Estados eligen sus propios niveles de protección previendo la amenaza de disputa (en caso de que un miembro aplique un estándar más restrictivo al comercio de lo necesario para alcanzar el nivel de protección requerido) que se presenta como un incentivo hacia el alineamiento de los estándares domésticos de seguridad alimentaria con los desarrollados por la Comisión del Codex Alimentarius. LINE FrIIS LINDNER, "Regulating Food Safety: The Power of Alignment and Drive towards Convergence", The European Journal of Social Science Research, 21 (2008), 133-143.

25 LIDNER explica que "la importancia atribuida a la OMC y su organización de referencia, la Comisión del Codex Alimentarius, aclara las restricciones institucionales inherentes al cuadro normativo internacional que gobierna la seguridad alimentaria a la vez que revela el poder indirecto de alineamiento tras esos estándares internacionales. Por otro lado, igualmente importantes son los estándares requeridos o a los que se adhiere por los socios comerciales clave": LINDNER, ob. cit., p. 133. 
La intención de armonizar la normativa alimentaria regional no es reciente, pudiendo rastrearse los primeros intentos en proyectos presentados durante el i Congreso Sudamericano de Química (Buenos Aires, 1924), cuando se propuso la redacción de un Codex Alimentarius Sudamericano ${ }^{26}$. El Código Latinoamericano de Alimentos fue aprobado en 1959, convirtiéndose en el primer código internacional de alimentos del mundo, previo al Codex Alimentarius que la FAO y la OMS sancionaran en 1962, el cual lo toma como base ${ }^{27}$.

Sin embargo, no será sino desde la década de 1990, debido a los grandes cambios en el régimen del comercio internacional de alimentos y a la celebración de acuerdos de integración regionales, que la normativa alimentaria experimentará grandes modificaciones en pos de las tendencias de armonización global y convergencia regional. Una de las más importantes fuentes de armonización son las resoluciones del Grupo Mercado Común del Mercosur.

El Grupo Mercado Común es el órgano ejecutivo del Mercosur y está coordinado por los Ministerios de Relaciones Exteriores. Se pronuncia a través de resoluciones que son obligatorias para los Estados Partes. El Tratado de Asunción (art. 13) permite al GMC constituir sub-grupos de trabajo (SGT) para el cumplimiento de sus cometidos. Estos subgrupos, inicialmente once, "cumplieron importantes funciones a los efectos de la coordinación de legislación durante la etapa de transición del Mercosur (1991-1995) [...] Con la posterior creación de la Comisión de Comercio de Mercosur, los SGT originales han sido objeto de una readecuación de funciones y estructura" ${ }^{28}$.

En materia sanitaria, se han logrado grandes avances ${ }^{29}$. En lo que interesa a este trabajo, cabe mencionar la labor del sGT n. ${ }^{\circ} 3$ de Reglamentos Técnicos

26 La Comisión estaba compuesta por dos delegados representantes de cada uno de los países adherido al evento (Dres. Coppetti y Peluffo, por Uruguay; Dres. Guggiari y Crovato por Paraguay; Dr. Ceriotti y Dr. Rumi por Argentina).

27 Carlos Grau, "Nuevo Codex Alimentarius de La Provincia de Buenos Aires", en Actas de trabalho, $7^{a}$ secção (presented at the $3^{\circ}$ Congresso Sul-americano de Chimica, Rio de Janeiro- São Paulo, 1937), 462-463.

28 Cfr.: [http://www.comercio.gob.ar/web/index.php?pag=193\&btn=163] Acceso: 21/06/2013. Los SGT actualmente son diecisiete (Anexo "Estructura del Grupo Mercado Común" de la Decisión CMG 12/2012): SGT n. 1 "Comunicaciones", sGT n. ${ }^{\circ} 2$ "Aspectos Institucionales", SGT n. 3 "Reglamentos Técnicos y Evaluación de la Conformidad", SGT n. 4 "Asuntos Financieros", SGT n. 5 "Transportes", SGT n. 6 "Medio Ambiente", SGT n. 7 "Industria", SGT n. 8 "Agricultura", SGT n. 9 "Energía", SGT n. ${ }^{\circ} 10$ "Asuntos Laborales, Empleo y Seguridad Social”, sGT n. 11 "Salud", sGT n. ${ }^{\circ} 12$ "Inversiones", sGT n. 13 "Comercio Electrónico", SGT n. 14 "Integración Productiva", SGT n. ${ }^{\circ} 15$ "Minería y Geología", SGT n. ${ }^{\circ} 16$ "Contrataciones Públicas", SGT n. 17 "Servicios". La Decisión enumera también Grupos de Trabajo (cinco) y una serie de Reuniones Especializadas (RES) y Reuniones Ad Hoc (RAH) como órganos del GMC.

29 Fundamentalmente mediante la comisión del SGT n. o 8 "Agricultura". SEBASTIÁn LEAVY and Francisco SÁEz, "Debilidades en la armonización de medidas sanitarias y fitosanitarias en el Mercosur", Densidades n. 5 (Buenos Aires, 2010), pp. 18-37 (p. 27). Esta comisión se encarga de la coordinación de políticas agrícolas y de posiciones en foros internacionales, de la adopción de medidas sanitarias y fitosanitarias (Manual de procedimiento zoosanitario en fronteras, nor- 
y Evaluación de la Conformidad, el cual tiene a cargo distintas comisiones específicas $^{30}$. Una de ellas se encarga de la cuestión de los alimentos ${ }^{31}$.

\section{LA REGULACIÓN DE LOS ADITIVOS ALIMENTARIOS}

La regulación de los aditivos alimentarios constituye una de las primeras preocupaciones de los organismos internacionales en materia alimentaria ${ }^{32}$. En el plano internacional, se destaca el Comité Mixto FAo/oms de Expertos en Aditivos Alimentarios, conocido por su acrónimo en inglés, JECFA (Joint Expert Committee on Food Aditives). Este Comité, que actualmente forma parte del sistema del Codex Alimentarius, se conformó en 1955. Es decir, existe desde antes de la creación del propio Codex $(1961)^{33}$. Desde entonces, el JECFA ha venido elaborando datos "que son ampliamente utilizados por los gobiernos, la industria y los centros de investigación [...] y las publicaciones basadas en sus actividades son reconocidas como referencias internacionales" ${ }^{\prime 4}$.

mas sobre cuarentena animal y vegetal, armonización de los sistemas de registro de productos fitosanitarios, etc.) y normas sobre semillas y productos vitivinícolas.

$30 \mathrm{El} \mathrm{sGT} \mathrm{n.}{ }^{\circ} 3$ del GMC tiene por objetivo "procurar la eliminación de los obstáculos técnicos al comercio y así, facilitar la libre circulación de los bienes y la integración regional entre los Estados Partes". El subgrupo se compone de coordinadores nacionales designados por cada Estado Parte. Tiene a su cargo las comisiones de Alimentos, Industria Automotriz, Metrología, Evaluación de la Conformidad, Productos Eléctricos y Gas Natural. Cfr.: [http://www.puntofocal. gov.ar/mercosur_sgt_subgrupo.htm]. Acceso: 15/04/2014.

31 Elabora normas de alcance general, como por ejemplo la Resolución GMC 80/96 (que establece las "Condiciones higiénico-sanitarias y buenas prácticas de fabricación para establecimientos elaboradores/industrializadores de alimentos") y otros grandes temas codificados, como el rotulado de alimentos (p. ej., Res. GMC 26/03, Reglamento Técnico Mercosur para la Rotulación de Alimentos Envasados); los aditivos y coadyuvantes del tecnología (p. ej., Res. GMC 11/06, Reglamento Técnico Mercosur sobre "Lista General Armonizada de Aditivos Alimentarios y sus Clases Funcionales"). También interviene en la elaboración de normativa sobre y envases, contaminantes y plaguicidas, productos procesados y productos in natura. [http://www.puntofocal. gov.ar/mercosur_sgt_alim.htm].Acceso 21/06/2013.

32 Los aditivos alimentarios son productos agregados a los alimentos para "mejorar su aspecto, textura, consistencia, color, sabor o aroma tornándolos más apetecibles, o para impedir su alteración, sin volverlos nocivos". También existen "aditivos involuntarios" o contaminantes, es decir, "sustancias de muy diversa naturaleza que pueden incorporarse a los alimentos en el curso de la cosecha de las materias primas, en el lavado, en la elaboración o en el envasado. Al ser ingeridos con el alimentos pueden provocar trastornos de distintas gravedad": Adolfo Leandro Montes, Bromatología (Eudeba, 1981), p. 6.

33 En 1953 el órgano rector de la oms declaró que "la utilización cada vez más amplia de sustancias químicas en la industria alimentaria representa un nuevo problema para la salud pública", y propuso llevar a cabo estudios al respecto, conjuntamente con la FAO. Cfr. FAO, ¿Qué es el Codex Alimentarius?, p. 10. Como resultado de uno de esos estudios, en 1955 la fao y la oms convocaron la primera Conferencia Mixta FAO/OMS sobre Aditivos Alimentarios, producto de la cual se creó el mencionado Comité Mixto FAO/oms de Expertos en Aditivos Alimentarios, JECFA.

34 FAO, ¿Qué es el Codex Alimentarius?, p. 26. 
El Codex Alimentarius y el Programa Conjunto Fao/oms sobre alimentos brindan a los Estados nacionales una guía oficial de referencia internacional para determinar sus regulaciones alimentarias, incluyendo criterios sobre la utilización de aditivos alimentarios ${ }^{35}$. Como vimos, más allá del derecho soberano de cada país a determinar su propia regulación, el Codex Alimentarius opera como un factor de armonización legislativa indirecta, en especial por dos razones. Por un lado, la legitimidad científica de los equipos de expertos que integran o asesoran a los distintos organismos, comités y reuniones del Codex, que cuentan con el reconocimiento de las distintas comunidades nacionales de expertos (los cuales suelen estar activamente involucrados en los procesos de diseño normativo en materia alimentaria). Por otro lado, desde la puesta en vigencia de la OMC en 1995 (que incluyó los AMSFS y AOTC) el impacto de las recomendaciones del JECFA se ha profundizado debido a la presunción de legitimidad de los parámetros del Codex Alimentarius (los cuales se considera que no representan un obstáculo al comercio internacional).

De allí la gran injerencia de estos estándares globales sobre las políticas alimentarias nacionales, especialmente de aquellos países que priorizan la apertura al comercio internacional de alimentos (exportación de materia prima, fundamentalmente). Como vimos, las medidas SFS adoptadas que se basan en esos estándares internacionales son consideradas legítimas; las que establezcan estándares más rigurosos, en principio, serán consideras ilegítimas barreras al comercio internacional (art. 2.2 AMSFS). El artículo 3.3 del Acuerdo permite medidas más elevadas pero requiere una justificación científica para esa protección extra. La justificación científica se explica en el artículo 5, según el cual se requiere que los desafíos a las medidas SFs estándares deben tener en cuenta "las técnicas de evaluación del riesgo elaboradas por las organizaciones internacionales competentes" ${ }^{36}$, ciertos factores económicos pertinentes ${ }^{37}$ y el "objetivo de reducir al mínimo los efectos negativos sobre el comercio" (art. 5.4 AMSFS).

35 Además del JEFCA, otras agencias nacionales o regionales de seguridad alimentaria son internacionalmente reconocidas por el prestigio de sus investigaciones y la trayectoria de sus regulaciones. En este sentido, cabe mencionar a la Autoridad Europea de Seguridad Alimentaria (EFSA, European Food Safety Authority) y la Administración de Alimentos y Medicamentos de Estados Unidos (FDA, Food \& Drug Administration).

36 Se establece que "al evaluar los riesgos, los Miembros tendrán en cuenta: los testimonios científicos existentes; los procesos y métodos de producción pertinentes; los métodos pertinentes de inspección, muestreo y prueba; la prevalencia de enfermedades o plagas concretas; la existencia de zonas libres de plagas o enfermedades; las condiciones ecológicas y ambientales pertinentes; y los regímenes de cuarentena y otros" (art. 5.2).

37 Los que implican "el posible perjuicio por pérdida de producción o de ventas en caso de entrada, radicación o propagación de una plaga o enfermedad; los costos de control o erradicación en el territorio del Miembro importador; y la relación costo-eficacia de otros posibles métodos para limitar los riesgos" (art. 5.3 AMSFS). 


\section{II.1. El alineamiento regional tras los estándares globales}

La regulación de los estándares relativos a los aditivos y otras sustancias agregadas a los alimentos en el Mercosur ilustra los modos en que se globalizan los parámetros internacionales pero a la vez presenta algunas particularidades que permiten vislumbrar formas de regulación alternativas a nivel regional.

En relación a la cuestión de los aditivos alimentarios, encontramos varias resoluciones del $\mathrm{GMC}^{38}$. Aquí nos concentraremos en lo establecido por la Resolución GMC 17/93, sobre Criterios de Mantenimiento de la Lista General de Aditivos Alimentarios Mercosur (la Lista General Armonizada de Aditivos Alimentarios y sus Clases Funcionales vigente se encuentra en el Reglamento Técnico de la Resolución GMC 11/06).

La Resolución GMC 17/93 reglamenta el procedimiento regional para mantener actualizada la lista de criterios admitidos (que la Res. GMC 11/06 toma de los parámetros establecidos por los mencionados organismos internacionales) obligando a los Estados parte a adoptar todas las medidas necesarias para dar cumplimento a esta norma (art. 2 Res. GMC 17/93). El Anexo de la Resolución regula diferencialmente el proceso de decisión según tres casos: inclusión, exclusión y "casos por excepción”.

La inclusión en la lista general procede respecto de aditivos propuestos por lo menos por un Estado Parte, a condición de que el aditivo en cuestión sea "admitido por el Codex Alimentarius o la CEE, pudiendo tomarse como información suplementaria la FDA" 39 .

La exclusión de aditivos alimentarios de la lista general se resuelve caso por caso y corresponde cuando "el Codex Alimentarius y la CEE resuelvan su eliminación".

Finalmente, se consideran casos por excepción todos aquellos pedidos de inclusión o de exclusión de la lista general de aditivos alimentarios por parte de algún Estado que no pueden encuadrarse en ninguno de los anteriores, siempre que se presenten "razones debidamente fundamentadas y respaldadas en estudios efectuados por organismos internacionalmente reconocidos". Estos organismos son expresamente mencionados: 1. la Agencia Internacional para la Investigación en Cáncer (IARC - International Agency for Research on Cancer $)^{40}$, 2. el mencionado JECFA del Codex Alimentarius, 3. el Instituto

38 Entre otras, cabe mencionar las resoluciones GMC 31/92, 17/93, 18/93 y 10/06 (Reglamento Técnico Mercosur sobre Aditivos Aromatizantes/Saborizantes) que establecen la definición y los principios fundamentales sobre el empleo de aditivos, ingredientes, coadyuvante de elaboración y contaminantes.

39 CEE significa Comunidad Económica Europea, actualmente, Unión Europea; y FDA es la agencia norteamericana de regulación de alimentos y medicamentos (Food and Drug Administration).

40 La Agencia Internacional de Investigación sobre el Cáncer (International Agency for Research on Cancer) pertenece a la oms. Se creó en mayo de 1965 a iniciativa de Francia y 
Nacional de Salud Pública y Protección Ambiental (Centro de Investigaciones del Hombre y el Ambiente) de Holanda (National Institute of Public Health \& Environmental Protection-RIVM-Research for Man \& The Environment $)^{41}$ y 4. el Centro Internacional de Información de Toxicología (ITIC, International Toxicology Information Center). El punto 3.4 de la Resolución GMC brinda este listado con los respectivos datos de contacto de dichos organismos. Todos ellos se localizan en la Unión Europea, en las ciudades de Lyon (Francia), Ginebra (Suiza), Bilthoven (Holanda) y San Sebastián (España).

Es decir, la inclusión o exclusión de sustancias de la lista necesita la legitimación de los estándares elaborados por los organismos internacionales. La resolución no prevé el caso de propuesta de uno o más Estado parte basándose exclusivamente en resultados de investigaciones endógenas. Incluso la incorporación de aditivos a la lista general en estos "casos excepcionales" a propuesta de algún Estado parte, también procede "basándose en estudios realizados por organismos reconocidos internacionalmente".

La inclusión de un aditivo "por excepción" corresponde a propuesta de algún Estado Parte y "su tratamiento se hará para cada aditivo, basándose en estudios realizados por organismos reconocidos internacionalmente, y su aprobación será por consenso (unanimidad)" (punto 3.2 Res. 17/93). La exclusión "por excepción" corresponde a solicitud de un Estado Parte, debiendo el mismo "presentar la documentación fundamentada y respaldada por algún organismo reconocido internacionalmente. En este caso la misma será aceptada sólo por consenso (unanimidad)" (punto 3.3 Res. 17/93).

En términos generales, podemos decir que la exclusión de aditivos del listado general corresponde automáticamente cuando el Codex Alimentarius y la CEE hayan resuelto su eliminación, mientras que la inclusión, si bien procede a pedido de parte, debe necesariamente estar apoyada por estudios de estos dos organismos internacionales (aunque suplementariamente se admiten también estudios de la FDA). Cuando no existan estos estudios, se admiten pedidos excepcionales, pero también deben estar avalados por alguna de las cinco entidades regulatorias extranjeras expresamente mencionadas.

tiene su sede en Lyon. Promueve y coordina la colaboración internacional en la investigación sobre esta enfermedad; propone y desarrolla estrategias científicas para su prevención y control. Reúne expertos en epidemiología, ciencias básicas y bioestadística para identificar las causas del cáncer. La IARC actualmente cuenta con veintiún países miembros. Cfr.: http://www.isciii.es/ ISCII/es/contenidos/fd-internacional/fd-colaboracion-internacional/iarc.shtml

41 El Instituto holandés para la Salud Pública y el Ambiente (conocido por su acrónimo en holandés RIVM, Rijksinstituut voor Volksgezondheid en Milieu) es un instituto de investigaciones y agencia independiente en la órbita del Ministerio de Salud, Bienestar y Deporte de Holanda. Lleva adelante tareas para la promoción de la salud pública y un ambiente de vida seguro, investigando y recolectando conocimiento de todo el mundo. Los resultados se utilizan para basar la formulación de políticas del gobierno holandés. El RIVm elabora una Base de Datos de la Composición de Alimentos (NEVO) que contiene datos sobre la composición de alimentos y preparaciones con alimentos que usualmente consume la población del país. Cfr.: [http://www.rivm.nl/English/]. 
La decisión queda a cargo de la Comisión de Alimentos Industrializados del Subgrupo de Trabajo n. 3 (sobre Normas Técnicas) del GMC.

Es decir, el mantenimiento de una sustancia en la lista general de aditivos alimentarios de la región se resuelve en función de un re-examen del conocimiento producido en espacios organizados y coordinados por organismos internacionales y/o agencias de países centrales. No está previsto el supuesto de necesidad de producción de nuevo conocimiento sobre la materia como base para adoptar modificaciones normativas.

Los criterios para el mantenimiento del listado de aditivos alimentarios ejemplifica el proceso de armonización legislativa regional alineado tras la regulación global (sin dejar posibilidad para un impulso hacia la convergencia regional). "Al no existir un comité científico que permita garantizar la evaluación propia" en la región, ambos procesos regulatorios (regional y global) se acoplan. De este modo, las aprobaciones de aditivos alimentarios se basan "en lo que dictaminan los comités de expertos científicos de la UE y de Ee.uU." 42 .

Esta dinámica se explica por la expresa intención de armonizar los Reglamentos Técnicos para tender a la eliminación de "los obstáculos que se generan por diferencias en la Reglamentaciones Nacionales vigentes" y poder contribuir a "ubicar los productos de los Estados Partes en el marco de la globalización" (considerandos de la Resolución GMC 11/2006 que actualiza la Lista General Armonizada de Aditivos Alimentarios y sus Clases Funcionales).

La debilidad de la región frente a esos procesos de globalización viene dada por una serie de problemas que en general se han señalado en los regímenes regulatorios de los alimentos en los países en desarrollo: superposición de competencia, dificultad del cumplimiento de sus reglas y de cooperación multisectorial, fragmentación regulatoria ${ }^{43}$ y falta de capacidad estructural (de recursos humanos, financieros e institucionales) para llevar adelante investigaciones científico técnicas que permitan discutir en el seno de los organismos regulatorios internacionales una posición propia adoptada según las necesidades particulares de la región.

Según la FAO, los sistemas legislativos alimentarios en los países en desarrollo están menos organizados, son menos comprensivos y menos efectivos que los sistemas de los países desarrollados, porque si bien las normas de inocuidad de los alimentos de los países en desarrollo "pueden equipararse de hecho a las normas internacionales", son "menos efectivas debido a la falta de capacidad institucional y técnica para vigilar y asegurar su cumplimiento.

42 Marina TorResani, "Edulcorantes" (Sociedad Argentina de Nutrición, 2012), en: [http:// www.sanutricion.org.ar/content/docs/pdf/2012/actualizacionesonline/edulcorantes.pdf].

43 Van Der Meulen, "Development of Food Legislation around the World: Concluding Observations", en Ensuring global food safety: exploring global harmonization, ed. by CHRISTINE BOISROBERT, 2010, pp. 63-66. 
Una infraestructura técnica insuficiente, en cuanto a laboratorios alimentarios, recursos financieros y humanos, marcos reglamentarios y jurídicos nacionales, capacidad de aplicación, gestión y coordinación, reduce la capacidad de hacer frente a estas dificultades", advirtiendo que estas deficiencias, "además de ser una amenaza para la salud pública, pueden reducir también el acceso comercial a los mercados alimentarios mundiales" 44 .

Los países desarrollados cuyos estándares se consideran modélicos, cuentan con la infraestructura necesaria para movilizar investigaciones precisas y desarrollar el conocimiento que permita poner en cuestión los parámetros estandarizados y utilizarlos estratégicamente como herramientas de protección de sus economías deteniendo las exportaciones, cuestionando en los foros internacionales la actuación de otros Estados competidores ${ }^{45}$. Estos países tienen un margen mayor para construir sus propias agendas y apartarse, si lo consideran necesario, de las recetas globales. Mientras que la mayoría de los países en desarrollo no logran participar en el proceso de establecimiento de estándares alimentarios del Codex, el cual produce y modifica los mismos ${ }^{46}$.

\section{II.2. Una excepción a la regla de alineamiento tras los estándares globales: las especies botánicas de origen regional}

En este punto introducimos en el análisis la regulación del Reglamento Técnico Mercosur sobre Aditivos Aromatizantes/Saborizantes (Res. GMC 10/06) para ser aplicado a los "aditivos aromatizantes/saborizantes que son producidos y comercializados en los territorios de los Estados Partes del Mercosur, al comercio entre ellos y a las importaciones de extrazona" (punto 1$)^{47}$.

El reglamento toma como lista de base o de referencia para los aromatizantes/saborizantes a todos los componentes "con uso aprobado, como mínimo, por una de las entidades: JECFA, UE (CoE), FDA o FEMA" (punto 5.1.1 Anexo Res. 10/06 GMC) y establece un listado de fuentes bibliográficas re-

44 "Estrategia de la FAO relativa al enfoque de calidad e inocuidad de los alimentos basado en la cadena alimentaria: documento marco para la formulación de la futura orientación estratégica", FAO, doc. COAG/2003/5 (2003), en: [http://www.fao.org/docrep/MEETING/006/ Y8350s.HTM].

45 Si se observan las partes litigantes en los casos de arbitraje internacional vinculado a la aplicación del AMSFS más relevantes, se trata de países o regiones centrales (Unión Europea y Estados Unidos, en numerosos casos); cfr.: [http://www.wto.org/spanish/tratop_s/dispu_s/ dispu_agreements_index_s.htm?id=A19\#selected_agreement].

46 Ching-Fu Lin, "Global Food Safety: Exploring Key Elements for an International Regulatory Strategy", Virginia Journal of International Law, 51 (2011), 637.

47 Este reglamento establece una serie de definiciones y clasificaciones, designaciones y formas de presentación (puntos 2, 3, y 4). Al igual que la regulación del Listado General de Aditivos Alimentarios (Res. GMC 17/93) se dicta a los fines de eliminación de obstáculos generados por las diferentes reglamentaciones nacionales vigentes y considerando que ese reglamento "contempla las solicitaciones de los Estados Partes". 
conocidas a las que deben responder los requisitos de identidad, pureza y demás especificaciones ${ }^{48}$.

Las únicas sustancias que escapan a la estandarización global son las "Especies Botánicas de Origen Regional" (punto 5.2 Anexo Res. 10/06 GMC) que por su especificidad son listadas individualmente en la resolución ${ }^{49}$. Todas ellas, así como sus principios activos aromatizantes/saborizantes, son comprendidas en la lista de base con las limitaciones establecidas. Sucede que, al carecer de referentes internacionales, se hace necesario construir criterios detallados para la actualización de esta lista de especies botánicas de origen regional. El reglamento regula el uso de estos componentes según cuatro categorías de especies botánicas de origen regional:

- N1. Frutas y hortalizas, o partes de las mismas, consumidas como alimentos. El uso de estos componentes en condiciones habituales de consumo no tiene restricciones.

- N2. Plantas y/o partes de las mismas, incluyendo hierbas, especias y condimentos comúnmente agregados a las comidas, en pequeñas cantidades. Se acepta su uso con posible limitación de algún principio activo en el producto final.

- N3. Plantas y/o partes de las mismas que, en vista de su larga historia de uso sin evidencia de efectos adversos agudos, son aceptadas temporariamente para su uso en ciertas bebidas y alimentos, en su forma tradicional. Se trata

48 El FEMA (Flavor and Extract Manufacturers Association of America Expert Panel) de Estados Unidos, fundado en 1909, es la asociación nacional de la industria de los saborizantes y aromatizantes norteamericana. "Trabajan con legisladores para asegurarse de que las necesidades de sus miembros y de los consumidores sean continuamente atendidas. El FEMA está comprometido con la garantía de un abastecimiento de sustancias saborizantes seguras". Cfr.: https:// www.femaflavor.org/about-fema Acceso: 15/04/2014. Esta asociación, junto con las siguientes entidades, son reconocidas como fuentes bibliográficas: CAS -"Chemical Abstracts Service", American Chemical Society (Washington, D.C); EFSA - European Food Safety Authority; FAO/ wHo Codex Alimentarius Standards; FCC - "Food Chemical Codex", National Academy Press (Washington, D.C.); FENAROLI - "Handbook of Flavor Ingredients" (CRC Publishing Co., Boca Raton, FL); IOFI - International Organization of the Flavor Industry, "Code of Practice of the Flavor Industry; JECFA, Sumary of Evaluations Performed by Joint FAO/wHO Expert Committee on Food Additives; Steffen Arctaner, "Perfume and Flavor Materials Natural Origin", 1994, Allured Publishing Co, USA; The Merck Index; TNO - Nutrition and Food Research Institute (The Netherlands) Volatile Compounds in Food Qualitative and Quantitative-Data; usa Code of Federal Regulation - CFR / Food and Drug Administration - FDA; y la Farmacopea Nacional de los Estados Partes.

49 Las especies botánicas de origen regional son: $a$ ) Calafate (Michay) - Berberis buxifolia Lam, Berberis heterophylla Juss, Berberis darwinii Hook. b) Canchalagua - Centarium cachanlahuen (Moll) Robinson. c) Carqueja - Baccharis articulata (Lamarck) Pers. Baccharis crispa Sprengel. d) Incayuyo - Lippia integrifolia (Griseb) Hieron. e) Lucera - Pluchea sagittalis (Lamarck) Cabrera.f) Maqui - Aristotelia chilensis (Molina) Stuntnz (sinônimo: Aristotelia macqui L'Herit). g) Marcela - Achyrocline satureioides (Lamarck) D.C. h) Peperina - Minthostachsys mollis (H.B.) Gris. i) Poleo - Lippia turbinata Griseb.j) Vira-vira - Gnaphalium cheiranthifolium Lam. k) Zarsaparrilla - Smilax campestris Gris. 
de casos para los cuales "las informaciones disponibles son insuficientes para determinar adecuadamente su potencial toxicidad a largo plazo". El uso de saborizantes/aromatizantes de esta categoría puede estar limitado.

- N4. Plantas y/o partes de las mismas que son utilizadas actualmente como aromatizantes/saborizantes y que no pueden clasificarse en las categorías N1, N2 o N3 debido a que la información es insuficiente.

El Reglamento detalla los requisitos de evaluación de las especies botánicas de cada categoría y el procedimiento para su inclusión en la lista. Las especies de las categorías N1 y N2 son incorporadas a la lista de base sin requisitos adicionales. Las N3 son incorporadas "temporariamente a la lista de base, luego de una evaluación de seguridad y aprobación por la autoridad competente del Estado Parte". Deben cumplir también otros requisitos: "registrar una larga historia de uso en la elaboración de bebidas y alimentos, considerando nombre(s) popular(es), parte de la planta y forma de preparación que es utilizada", tener una "identificación botánica inequívoca de la especie y de sus variedades, con depósito de ejemplares en herbarios de referencia", y su uso debe estar de acuerdo con la limitación de principios activos en el producto final previsto en la resolución. Se establece la temporalidad de la inclusión en la lista hasta la realización de "estudios de evaluación que comprueben su seguridad" 50 .

Finalmente, la utilización de las especies de la categoría N4 no será permitida "hasta que sean obtenidas las informaciones sobre su identidad y calidad mediante la identificación botánica inequívoca de la especie y de sus variedades, con depósito de ejemplares en herbarios de referencia; y estudios farmacognósticos y fitoquímicos de los principales componentes, determinación de principios activos tóxicos, metodologías de análisis, estudios toxicológicos de efectos agudos y crónicos".

La decisión acerca de la autorización del consumo de especies aromatizantes/ saborizantes regionales pone a los Estados en la situación de tener que proceder a la realización de estudios endógenos y genera la necesidad de reglamentar un procedimiento también propio, ya que no existen pautas internacionales predeterminadas (por la localización limitada de las especies) que limiten o condicionen las resoluciones al respecto. Sin los condicionamientos de un mercado global, los países de la región recaban el conocimiento científico local necesario para basar las medidas que libremente adoptan al respecto.

50 Aclara que se trata de estudios fármacognósticos y fitoquímicos de los principales componentes, determinación de principios activos tóxicos y metodologías de análisis; estudios toxicológicos de efectos agudos y estudios de corto plazo que puedan, incluso, indicar la necesidad de estudios de largo plazo para la evaluación de efectos crónicos. 


\section{CONCLUSIONES}

En materia de uso de aditivos alimentarios, la regulación de la región Mercosur se alinea tras el sistema global de regulación alimentaria, especialmente las recomendaciones de la agencia global de fao/oms, el Codex Alimentarius (debido al impacto de las evaluaciones de su Comité de Expertos sobre Aditivos Alimentarios -JECFA- en las resoluciones del GMC). Los objetivos de remoción de obstáculos al comercio, para facilitar la exportación de los recursos locales, determinan esta regla de alineamiento generalizado. La fuerza de la globalización de las listas positivas consensuadas en los foros intergubernamentales neutraliza la capacidad de regulación autónoma de que en principio goza cualquier Estado.

La aprobación de aditivos alimentarios en los países del Mercosur se basa en el dictamen de comités de expertos científicos extranjeros. En el marco regional del Mercosur no se prevé, en principio, el supuesto de producción de nuevo conocimiento sobre aditivos alimentarios como base para la introducción de modificaciones a la normativa general. Como excepción, vimos que los países de la región libremente adoptan decisiones sobre la autorización de especies botánicas regionales. En este caso los países de la región se enfrentan a la necesidad de contar con información suficiente para basar las medidas al respecto. Ante la carencia de parámetros internacionales que limiten o condicionen sus resoluciones, deben también reglamentar un procedimiento con características propias adecuadas a estas especies particulares.

Es fundamental para los Estados en desarrollo contar con infraestructura propia para realizar evaluaciones de riesgos que les permita adoptar medidas fitosanitarias que consideren necesarias, incluso si esto desafía los estándares globales. El logro de este objetivo no puede depender totalmente de la "asistencia" que está previsto brinden los Estados desarrollados y los organismos internacionales, la cual no puede esperarse sea por fuera de los marcos propios trazados para despejar de obstáculos el comercio internacional de alimentos reforzando la división internacional del trabajo en materia alimentaria. La asistencia de los organismos internacionales se limita al fomento de la tecnología estándar reproductiva del modelo propuesto y del entrenamiento de los aparatos estatales de control $-\mathrm{y}$, a través de ellos, del sector privado- en torno a las condiciones necesarias para la aplicación eficaz de la regulación global.

No será posible, con los recursos globalizados, generar herramientas para cuestionar estos estándares. Es necesario avanzar en el proceso de integración construyendo un conocimiento endógeno y capacidades estatales de aplicación y control suficientes para aplicar una legislación regional pensada a la medida de las necesidades locales de desarrollo y no (o al menos, no solamente) de las exigencias de un mercado globalizado de alimentos. 
Hacia el alineamiento global en la armonización regional: la regulación de los aditivos ... 201

\section{BIBLIOGRAFÍA}

Boutrif, E. "The New Role of Codex Alimentarius in the Context of wto/sps Agreement", en Food Control 14 (2003), 81-88.

Charnovitz, S. "Improving the Agreement on Sanitary and Phytosanitary Standards", en Trade, Environment, and the Millenium, Overseas Development Council, 2000, 171.

FaO. ¿Qué es el Codex Alimentarius?, Roma, FaO, 2006, Web. 15 nov. 2012.

FAo. Directrices en Materia de Legislación Alimentaria (nuevo Modelo de Ley de Alimentos para Países de Tradición Jurídica Romano-Germánica), FAO, 2006.

Grau, C. "Nuevo Codex Alimentarius de la Provincia de Buenos Aires", Actas de Trabalho, $7^{a}$ Secção, vi, Rio de Janeiro-São Paulo, 1937, 462-463.

Jukes, D. "The Role of Science in International Food Standards", Food Control 11.3 (2000), 181-194.

Larach, M. El Acuerdo sobre la Aplicación de Medidas Sanitarias y Fitosanitarias: Contenido y alcance para América Latina y el Caribe, Santiago de Chile, ONU- CEPAL, 2003. Comercio Internacional 39.

Leavy, S. y SAez, F. "Debilidades en la armonización de medidas sanitarias y fitosanitarias en el Mercosur", en Densidades n. . 5, 2010, 18-37.

Lin, C. "Global Food Safety: Exploring Key Elements for an International Regulatory Strategy", Virginia Journal of International Law 51.3 (2011), 637.

Lindner, L. "Regulating Food Safety: The Power of Alignment and Drive towards Convergence", The European Journal of Social Science Research 21.2 (2008), 133-143.

Livermore, M. "Authority and Legitimacy in Global Governance: Deliberation, Institutional Differentiation, and the Codex Alimentarius", New York University Law Review 81 (2006), 766-801.

Mansour, M. "One World for All: International Harmonization of Food Regulations. Codex Alimentarius and the Treatment of Foods Deriver from Biotechnology as a Case Study", Journal of Food Science 69.4 (2004).

Montes, A. Bromatología, Eudeba, 1981.

Torresani, M. "Edulcorantes", 2012, Web, 19 de diciembre de 2012.

Van Der Meulen, B. "Development of Food Legislation around the World: Concluding Observations", Ensuring Global Food Safety: Exploring Global Harmonization, Christine Boisrobert, 2010, 63-66.

Van Der Meulen, B. "The Global Arena of Food Law: Emerging Contours of a MetaFramework", Erasmus Law Review 3.4 (2010), 217-240.

Veggeland, F. y Borgen, S. "Negotiating International Food Standards: The World Trade Organization's Impact on the Codex Alimentarius Commission", Governance 18.4 (2005), 675-708. 
Winickoff, Davis, E. y Bushey, D. "Science and Power in Global Food Regulation: The Rise of the Codex Alimentarius", Science, Technology, \& Human Values 35.3 (2010), 356-381.

Wirth, D. "The Role of Science in the Uruguay Round and NAFTA Trade Disciplines", Cornell International Law Journal 27 (1994), 817-859. 